\title{
Crystal structure of ammonium [monozinco-monoboro-diphosphate], $\mathrm{NH}_{4}\left[\mathrm{ZnBP}_{2} \mathrm{O}_{8}\right]$
}

\author{
R. Kniep*, G. Schäfer and H. Borrmann
}

Max-Planck-Institut für Chemische Physik fester Stoffe, Pirnaer Landstr. 176, D-01257 Dresden, Germany

Received January 7, 2000, CSD-No. 409477

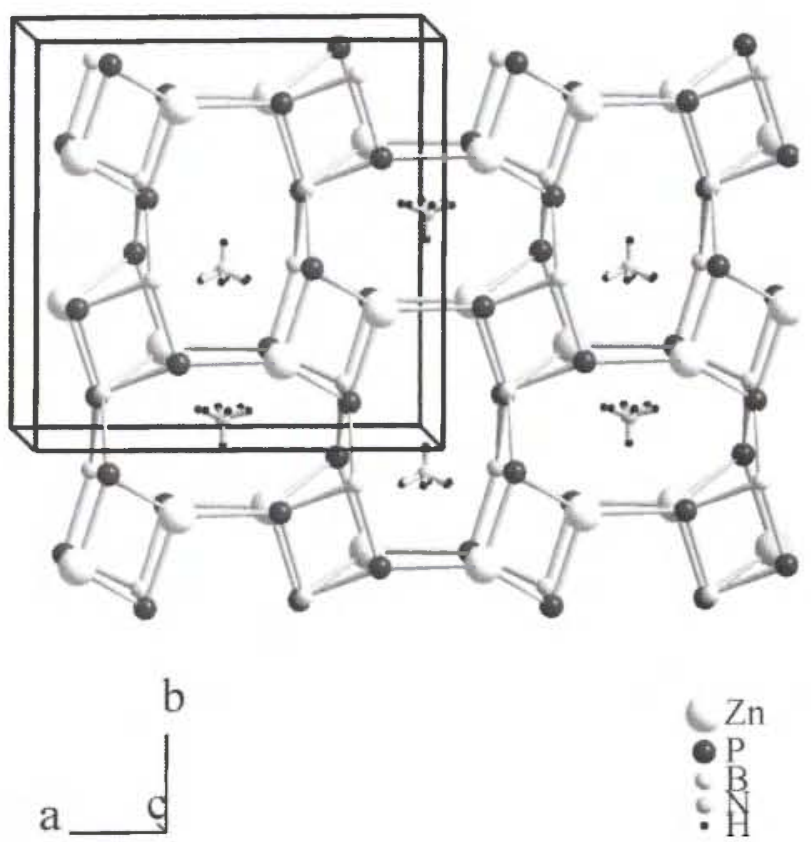

\section{Discussion}

The crystal structure of $\mathrm{NH}_{4}\left[\mathrm{ZnBP}_{2} \mathrm{O}_{8}\right]$ (anhydrous borophosphate, $\mathrm{B}: \mathrm{P}=0.5[1])$ contains an anionic framework $\left[\mathrm{ZnBP}_{2} \mathrm{O}_{8}\right]^{-}$, which is formed by alternating zincate, borate and phosphate tetrahedra sharing common corners. The ammonium compound is an isotype of $\mathrm{K}$ [ $\left.\mathrm{ZnBP}_{2} \mathrm{O}_{8}\right]$ [2]. The protons $\mathrm{H} 1, \mathrm{H} 2$, $\mathrm{H} 4, \mathrm{H} 6, \mathrm{H} 7$ and $\mathrm{H} 8$ of the ammonium cations are disordered over two different positions. Bond lenghts and angles within the anionic partial structure are consistent with related borophosphates (see $[1,2]$ and refs. herein). A second polymorph of $\mathrm{NH}_{4}\left[\mathrm{ZnBP} 2 \mathrm{O}_{8}\right]$ with a gismondine type framework is also known [2].

Table 1. Data collection and handling.

\begin{tabular}{|c|c|}
\hline Crystal: & $\begin{array}{l}\text { colourless plate, } \\
\text { size } 0.05 \times 0.125 \times 0.25 \mathrm{~mm}\end{array}$ \\
\hline Wāvêlēngth: & Mo $K_{\alpha}$ rádiation $(0.71073 \AA)$ \\
\hline$\mu:$ & $39.51 \mathrm{~cm}^{-1}$ \\
\hline Diffractometer, scan mode: & $\begin{array}{l}\text { Rigaku AFC7 CCD, } \Phi \text {-scan } 360^{\circ} \text {, } \\
60^{\circ} \text { - } \omega \text {-scan at } \chi=90^{\circ}, 0.5^{\circ} \text { steps with } 40 \mathrm{~s} \\
\text { exposure time per step, detector distance: } \\
35 \mathrm{~mm}, 2 \theta \text { offset: } 10^{\circ}\end{array}$ \\
\hline $2 \theta_{\max }:$ & $59.68^{\circ}$ \\
\hline$N(h k l)_{\text {measured }}, N(h k l)_{\text {unique: }}$ & 8548,1649 \\
\hline Criterion for $I_{\text {obs }}, N(h k l)_{\mathrm{gt}}$ : & $I_{\text {obs }}>2 \sigma\left(I_{\text {obs }}\right), 1580$ \\
\hline$N(\text { param })_{\text {refined: }}$ & 135 \\
\hline Programs: & $\begin{array}{l}\text { SHELXS-97 [3], SHELXS-97 [4], } \\
\text { DIAMOND [5] }\end{array}$ \\
\hline
\end{tabular}

\begin{abstract}
$\mathrm{BH}_{4} \mathrm{NO}_{8} \mathrm{P}_{2} \mathrm{Zn}$, monoclinic, $C 12 / c \mathrm{l}$ (No. 15), $a=12.848(3) \AA$, $b=12.896(3) \AA, c=8.519(1) \AA, \beta=91.024(3)^{\circ}, V=1411.2 \AA^{3}$, $Z=8, R_{\mathrm{gt}}(F)=0.040, w R_{\text {ref }}\left(F^{2}\right)=0.073, T=293 \mathrm{~K}$.
\end{abstract}

\section{Source of material}

$\mathrm{NH}_{4}\left[\mathrm{ZnBP} 2 \mathrm{O}_{8}\right]$ was prepared by solvothermal reaction (with ethylene glycol) of $0.700 \mathrm{~g} \mathrm{ZnO}, 0.298 \mathrm{~g} \mathrm{~B}_{2} \mathrm{O}_{3}, 2.264 \mathrm{~g}$ $\left(\mathrm{NH}_{4}\right)_{2} \mathrm{HPO}_{4}$ and $5 \mathrm{ml} 85 \% \mathrm{H}_{3} \mathrm{PO}_{4}$ (molar ratio: $2: 1: 4$, gel, $\mathrm{pH}=$ $1.5)$ in a teflon autoclave at $438 \mathrm{~K}$.

\section{Experimental details}

Refinement with restraints: $\mathrm{N}-\mathrm{H}$ distances to $0.86 \AA$, $\mathrm{H}-\mathrm{N}-\mathrm{H}$ angle to approximately the tetrahedral angle and $U_{\text {iso }}(\mathrm{H})=$ $1.2 \times U_{\text {iso }}(\mathrm{N})$
Table 2. Atomic coordinates and displacement parameters (in $\AA^{2}$ ).

\begin{tabular}{|c|c|c|c|c|c|c|}
\hline Atom & Site & & $x$ & y & $z$ & $U_{\text {iso }}$ \\
\hline$N(1)$ & $4 e$ & & 0 & $0.0346(3)$ & $1 / 4$ & $0.0200(9)$ \\
\hline$H(1)$ & $8 f$ & 0.5 & $0.052(5)$ & $0.057(2)$ & $0.21(1)$ & 0.024 \\
\hline $\mathrm{H}(2)$ & $8 f$ & 0.5 & $-0.01(1)$ & $0.056(2)$ & $0.344(4)$ & 0.024 \\
\hline $\mathrm{H}(3)$ & $4 e$ & & 0 & $-0.032(2)$ & $1 / 4$ & 0.024 \\
\hline $\mathbf{H}(4)$ & $8 f$ & 0.5 & $-0.056(6)$ & $0.054(2)$ & $0.20(1)$ & 0.024 \\
\hline $\mathbf{N}(2)$ & $4 e$ & & 0 & $0.3981(3)$ & $1 / 4$ & $0.025(1)$ \\
\hline$H(5)$ & $4 e$ & & 0 & $0.463(2)$ & $1 / 4$ & 0.03 \\
\hline$H(6)$ & $8 f$ & 0.5 & $-0.050(7)$ & $0.373(2)$ & $0.19(1)$ & 0.03 \\
\hline$H(7)$ & $8 f$ & 0.5 & $0.058(6)$ & $0.374(2)$ & $0.21(2)$ & 0.03 \\
\hline$H(8)$ & $8 f$ & 0.5 & $-0.01(1)$ & $0.372(2)$ & $0.343(4)$ & 0.03 \\
\hline
\end{tabular}

\footnotetext{
* Correspondence author (e-mail: schaefer@cpfs.mpg.de)
} 
Table 3. Atomic coordinates and displacement parameters (in $\AA^{2}$ ).

\begin{tabular}{|c|c|c|c|c|c|c|c|c|c|c|}
\hline Atom & Site & $x$ & $y$ & $z$ & $U_{11}$ & $U_{22}$ & $U_{33}$ & $U_{12}$ & $U_{13}$ & $U_{23}$ \\
\hline $\operatorname{Zn}(1)$ & $8 f$ & $0.12436(3)$ & $0.19147(3)$ & $0.56717(4)$ & $0.0121(2)$ & $0.0130(2)$ & $0.0114(2)$ & $-0.0007(2)$ & $-0.0003(1)$ & $-0.0007(1)$ \\
\hline $\mathrm{B}(1)$ & $8 f$ & $0.3072(3)$ & $0.1082(3)$ & $0.0743(4)$ & $0.012(2)$ & $0.010(2)$ & $0.010(2)$ & $0.003(1)$ & $0.000(1)$ & $-0.001(1)$ \\
\hline$P(1)$ & $8 f$ & $0.30153(6)$ & $0.08082(6)$ & $0.40616(9)$ & $0.0111(4)$ & $0.0096(4)$ & $0.0086(4)$ & $0.0006(3)$ & $-0.0006(3)$ & $0.0010(3)$ \\
\hline $\mathrm{P}(2)$ & $8 f$ & $0.37061(6)$ & $0.31392(6)$ & $0.06278(9)$ & $0.0088(4)$ & $0.0092(3)$ & $0.0100(4)$ & $-0.0003(3)$ & $-0.0016(3)$ & $0.0010(3)$ \\
\hline$O(1)$ & $8 f$ & $0.1384(2)$ & $0.3348(2)$ & $0.5069(3)$ & $0.023(1)$ & $0.013(1)$ & $0.020(1)$ & $-0.0034(9)$ & $-0.007(1)$ & $0.0016(9)$ \\
\hline$O(2)$ & $8 f$ & $0.1597(2)$ & $0.4752(2)$ & $0.0308(2)$ & $0.017(1)$ & $0.012(1)$ & $0.016(1)$ & $-0.0023(9)$ & $0.0034(9)$ & $-0.0050(9)$ \\
\hline$O(3)$ & $8 f$ & $0.1856(2)$ & $0.0941(2)$ & $0.4205(3)$ & $0.013(1)$ & $0.020(1)$ & $0.014(1)$ & $0.0019(9)$ & $-0.0009(9)$ & $-0.0039(9)$ \\
\hline$O(4)$ & $8 f$ & $0.1963(2)$ & $0.1300(2)$ & $0.0655(2)$ & $0.012(1)$ & $0.019(1)$ & $0.012(1)$ & $0.0029(9)$ & $-0.0008(9)$ & $0.0025(9)$ \\
\hline$O(5)$ & $8 f$ & $0.3281(2)$ & $0.3362(2)$ & $0.2218(3)$ & $0.022(1)$ & $0.023(1)$ & $0.009(1)$ & $0.005(1)$ & $0.0001(9)$ & $-0.0013(9)$ \\
\hline$O(6)$ & $8 f$ & $0.3359(2)$ & $0.0767(2)$ & $0.2342(3)$ & $0.016(1)$ & $0.031(1)$ & $0.009(1)$ & $0.008(1)$ & $0.0017(9)$ & $0.005(1)$ \\
\hline$O(7)$ & $8 f$ & $0.3708(2)$ & $0.1964(2)$ & $0.0245(3)$ & $0.017(1)$ & $0.011(1)$ & $0.022(1)$ & $-0.0012(9)$ & $0.007(1)$ & $-0.0009(9)$ \\
\hline$O(8)$ & $8 f$ & $0.4802(2)$ & $0.3528(2)$ & $0.0392(3)$ & $0.008(1)$ & $0.017(1)$ & $0.026(1)$ & $-0.0020(9)$ & $-0.0037(9)$ & $0.003(1)$ \\
\hline
\end{tabular}

Acknowledgments. We thank the Pinguin-Stiftung (Düsseldorf) and the Fonds der Chemischen Industrie for supporting this work.

\section{References}

1. Kniep, R.; Engelhardt, H.; Hauf, C.: A First Approach to Borophosphate Structural Chemistry. Chem. Mater. 10 (1998) 2930-2934.
2. Kniep, R.; Schäfer, G.; Engelhardt, $\mathrm{H}$; Boy, I.: $\mathrm{K}\left[\mathrm{ZnBP}_{2} \mathrm{O}_{8}\right]$ und $\mathrm{A}\left[\mathrm{ZnBP} \mathrm{O}_{8}\right]\left(\mathrm{A}=\mathrm{NH}_{4}{ }^{+}, \mathrm{Rb}^{+}, \mathrm{Cs}^{+}\right)$: Zinkoborophosphate als neue Klasse von Verbindungen mit Tetraeder-Gerüststrukturen. Angew. Chem. 111 (1999) 3857-3861, Angew. Chem. Int. Ed. 38 (1999) 3642-3644.

3. Sheldrick, G. M.: SHELXS-97-2, Program for the Solution of Crystal Structures. University of Göttingen, Germany 1997.

4. Sheldrick, G. M.: SHELXL-97-2, Program for Crystal Structure Refinement. University of Göttingen, Germany 1997.

5. Brandenburg, K.: Diamond (Version 2.1a). Crystal Impact GbR, Germany 1996-1999. 